\title{
Prescription Drug Use and Polypharmacy Among Medicaid- Enrolled Adults with Autism: A Retrospective Cross-Sectional Analysis
}

\author{
Rini Vohra $\left(\mathbb{D} \cdot\right.$ Suresh Madhavan $^{2} \cdot$ Usha Sambamoorthi $^{2}$ - Claire StPeter ${ }^{3} \cdot$ \\ Susannah Poe ${ }^{4,5} \cdot$ Nilanjana Dwibedi $^{2} \cdot$ Mayank Ajmera $^{6}$
}

Published online: 21 November 2016

(C) The Author(s) 2016. This article is published with open access at Springerlink.com

\begin{abstract}
Background A lack of gold standard treatment for autism spectrum disorders (ASD), no clear ASD management guidelines, and lack of evidence-based pharmacological interventions other than aripiprazole and risperidone elevate the risk of off-label prescribing and adverse effects among individuals with ASD, more so among adults.

Objective The aim of this study was to identify and compare the types of prescription drug use, rates of polypharmacy, and characteristics associated with polypharmacy among adults with and without ASD in a retrospective cross-sectional analysis of a three-state Medicaid Analytic eXtract database (2000-2008).
\end{abstract}

Electronic supplementary material The online version of this article (doi:10.1007/s40801-016-0096-z) contains supplementary material, which is available to authorized users.

Rini Vohra

rinievohra@gmail.com

1 Center for Medication Optimization Through Practice and Policy, UNC Eshelman School of Pharmacy, 2401 Kerr Hall, Chapel Hill, NC 27599, USA

2 Department of Pharmaceutical Systems and Policy, West Virginia University, 1 Medical Center Drive, Morgantown, WV 26506 9510, USA

3 Behavior Analysis Program Area, West Virginia University, 1124 Life Sciences Building, Morgantown, WV 26506-6040, USA

4 Department of Pediatrics, WVU School of Medicine, Klingberg Neurodevelopmental Center, West Virginia University, Morgantown, WV 26506 9510, USA

5 Autism/TRAIN, Center for Excellence in Disabilities (CED), Morgantown, WV, USA

6 RTI Health Solutions, Research Triangle Park, NC, USA
Methods Adults aged 22-64 years with ASD (ICD9-CM code: 299.xx) were propensity score-matched to 'no ASD' controls by age, sex, and race. General polypharmacy ( $\geq 6$ unique classes of prescription drugs in a year) and psychotropic polypharmacy ( $\geq 3$ unique prescription drug classes of psychotropic medications within a 90-day period) were the main study outcomes. Chi-square tests for rates, $t$ tests for mean number of claims, and multivariate logistic regressions for likelihood of prescription drug use and polypharmacy were run.

Results Annually, almost $75 \%$ of adults with ASD had $>20$ prescription drug claims compared with $33 \%$ of adults without ASD. Around $85 \%$ of adults with ASD used at least one psychotropic drug class compared with $42 \%$ of adults without ASD. Highly common psychotropics were antipsychotics $\left(66 \%_{\text {ASD }}\right.$ vs $\left.20 \%_{\text {noASD }}\right)$, anticonvulsants $\left(59 \%_{\text {ASD }}\right.$ vs $\left.20 \%_{\text {noASD }}\right)$, and anxiolytics/hypnotics/sedatives $\left(21 \%_{\text {ASD }}\right.$ vs $\left.11 \%_{\text {noASD }}\right)$. Other than psychotropics, many adults with ASD used medical prescription drugs such as antimicrobials (47\%), dermatologic agents (48\%), respiratory agents $(38 \%)$, gastrointestinal agents (31\%), alternative medications (25\%), antiparkinsonian agents (22.6\%), antihyperlipidemics/statins (7.3\%), and immunologics $(2.0 \%)$. Rates of general ( $48 \%_{\text {ASD }}$ vs $\left.32 \%_{\text {noASD }}\right)$ and psychotropic polypharmacy $\left(19 \%_{\mathrm{ASD}}\right.$ vs $\left.6 \%_{\text {noASD }}\right)$ were significantly higher in the ASD group.

Conclusion Prescription drug use and polypharmacy rates among adults with ASD are substantially higher than those in an age-, sex-, and race-matched cohort of adults without ASD. Adults with ASD frequently use therapeutic treatments other than psychotropics. Healthcare providers, who usually report low confidence in treating patients with ASD, should play an active role in constant monitoring of prescription drug use patterns and patient response to interventions. Prescribers and caregivers are encouraged to 
make decisions after weighing the benefits and risks associated with a pharmacological treatment. Further investigations into the common use of any alternative treatments that can affect a patient's response to core treatments should also be conducted.

\section{Key Points}

About half of adults with autism spectrum disorders (ASD) use six or more classes of prescription drugs in a year. Almost 20\% of adults with ASD use three or more classes of psychotropic drugs within a period of 90 days.

In addition to common use of psychotropics such as antipsychotics, antidepressants, and anxiolytics, many adults with ASD also use other medical treatments including dermatologic agents, antihyperlipidemics, gastrointestinal agents, antiparkinsonian agents, and immunologics.

There is a glaring need to build strong evidence for pharmacological interventions used by individuals with ASD, especially adults. More clinical trials including adults with ASD would help healthcare providers design optimum treatment regimens for their patients.

\section{Introduction}

Impaired communication and social interactions are some of the core symptoms of autism spectrum disorders (ASD). However, many individuals with ASD are also affected by challenging behavioral issues such as aggression, irritability, and self-injury [1-4] and multiple medical and psychiatric comorbidities [5-9]. Management of ASD and associated comorbidities may include an array of pharmacological interventions [10], which usually involves use of multiple prescription drugs [11-14]. To date, there are no prescription drugs that can treat core ASD symptoms, but the US Food and Drug Administration (FDA) approved risperidone (2006) and aripiprazole (2009) primarily for symptoms of irritability, hyperactivity, and aggression associated with ASD [15]. Pharmacological treatment is clinically essential or warranted in most cases, yet there are reasons why concurrent use of multiple prescription drugs (sometimes referred to as polypharmacy) [16-18] may be of concern. As the literature on prescription drug use and polypharmacy among adults with ASD is limited [19-21], studies including children, adolescents, and youth with ASD have been used to explain general prescription drug patterns among individuals with ASD.

\subsection{Lack of Evidence Base}

There is a lack of strong evidence for safety and efficacy of prescription drugs that are used off-label among individuals with ASD (where physicians often prescribe non-indicated medications in a trial-and-error fashion to help manage troublesome symptoms in patients, especially if other treatments are failing) [15]. An increasing number of studies are documenting the practice of off-label prescriptions by physicians to manage cases where the approved medications are either not available or not considered an adequate treatment option for individuals with ASD [22-24]. A Danish study using a national registry indicated a 4.7-fold increase in prescriptions of atomoxetine, methylphenidate, and dexamphetamine for children with ASD from 2003 to 2010 [24]. This practice is also observed with increased off-label use of psychotropic drugs including selective serotonin reuptake inhibitors (SSRIs) [12] for repetitive behaviors, stimulant medications for attentiondeficit hyperactivity disorder (ADHD)-type symptoms [25], and anti-epileptic medications such as valproate for behavioral symptoms in ASD [26].

\subsection{Effectiveness versus Side Effects}

Aripiprazole and risperidone display moderate effectiveness but are also associated with substantial adverse effects [15]. A systematic review by Dove et al. [20] on treatment of adults and adolescents (13-30 years) with ASD concluded there was moderate evidence for risperidone in controlling aggression and weak evidence for any other treatment. It is well known that risperidone therapy is associated with adverse events such as sedation, obesity, tardive dyskinesia, and side effects such as increased appetite, fatigue, drowsiness, dizziness, and drooling $[20,27]$. Aripiprazole use is also known to cause adverse effects such as significant weight gain, sedation, and sialorrhea [28-32]. In addition, a medical chart review highlighting the complexities of ASD pharmacological treatments by Adler et al. [33] showed that $40 \%$ of patients with ASD still required medication adjustment despite previous trials of risperidone and aripiprazole, or combinations of these.

Other prescribed medications such as antidepressants and mood stabilizers also have significant tolerability issues. A recent systematic review on clinical trials examining the efficacy of SSRIs (fluoxetine, fluvoxamine, fenfluramine, and citalopram) reported no significant improvements in ASD symptoms but rather an additional risk of harm [34]. Among children and adolescents with ASD, use of antidepressants was found to be associated with behavioral activation (hyperactivity and agitation), aggression, and suicidal ideation [35]. The long-term 
effects of prescription drug use and polypharmacy among individuals with ASD, especially the adult population, are unknown [10, 19]. Studies have shown that increasing age is significantly associated with greater likelihood of high prescription drug use among individuals with ASD [36-38], which necessitates further investigation on patterns of prescription drug use in the adult population.

The current study elaborates on prescription drug use patterns and types of polypharmacy among adults with ASD compared with adults without ASD. Most studies examining polypharmacy among individuals with ASD are limited to either pediatric populations and/or psychotropic polypharmacy $[28,31,33,34,36,38,39$, 40 , 41] (with rates from 10 to 20\%). A few studies among adults with ASD have examined psychotropic polypharmacy, but are restricted by small samples and clinical settings with a high chance of referral bias $[19,20]$. Previous studies have defined polypharmacy in many different ways [16, 17, 18, 42, 43, 44] and variability in polypharmacy rates has been observed with differences in operational definitions in administrative claims databases [45]. Some have defined it as concurrent use of multiple drugs with minor (two drugs) and major (more than four drugs) differentiations [16-18] and some have defined it as use of more drugs that are clinically indicated or excessive use of inappropriate drugs $[16,42]$. In the current study, we identified general polypharmacy as use of six or more unique prescription drug classes in a year [46], and psychotropic polypharmacy as concurrent use of three or more psychotropic drug classes within a 90-day period after the first prescription fill for a psychotropic drug [47]. We hypothesized that adults with ASD are at greater risk of polypharmacy than adults without ASD because of the lack of effectiveness evidence for this age group [15], a shortage of trained mental health professionals and facilities for optimum management of ASD cases [48], physician-reported lack of self-perceived competency and knowledge in treating adults with ASD [49-52], and no clarity on adult ASD treatment recommendations and evidence-based guidelines in the US.

Our study also describes the types of prescription drugs used among adults with ASD. A few studies with small sample sizes [21, 37, 53] have indicated that adolescents and adults with ASD are not only likely to use psychotropics (e.g., antipsychotics and antidepressants), but also commonly need and use non-psychotropic drugs (e.g., antilipemics, gastrointestinal medications, and other nonpsychotropic drugs) [21]. We expected that use of psychotropic and non-psychotropic prescription drugs would be more common among adults with ASD than adults without ASD, based on the high prevalence rates of medical comorbidities among adults with ASD found in a recent study by Croen et al. in 2015 [54], and other studies on psychotropic medication use in children with ASD [36, 38, 39].

We used a three-state Medicaid administrative claims database (Illinois [IL], New York [NY], and Texas [TX]; 2000-2008) to determine the rates of prescription drug use, general polypharmacy, and psychotropic polypharmacy among adults (22-64 years) with and without ASD in a 1:3 matched cohort (age, gender, and race). Although prescription drug coverage is an optional benefit under federal Medicaid law, all state Medicaid programs provide some type of coverage for outpatient pharmacy prescription drugs to eligible individuals. Medicaid plays a significant role in prescription drug coverage for individuals with mental health and substance abuse issues [55], and previous studies have utilized Medicaid claims databases to examine the rates of psychotropic polypharmacy among children with ASD [39, 40].

\section{Methods}

\subsection{Data}

Administrative health insurance claims data from three state Medicaid programs (IL, NY, and TX) were extracted from the 2000-2008 Centers for Medicare and Medicaid Services (CMS) Medicaid analytic eXtract (MAX) data files. We selected IL, NY, and TX MAX data because of lower managed-care penetration rates, greater fee-for-service enrollment during the study period, and large sample of enrollees with ASD in these state MAX files [56]. MAX offers separate data files that provide varied information on diagnosis codes, service use, demographic characteristics, and state of residence. Separate MAX data files received from CMS can be linked based on beneficiaries' unique identification numbers.

For the current study, we used the personal summary file (for information on Medicaid eligibility, enrollment type, and demographics), other therapy file (for information on two possible diagnoses, utilization of outpatient services such as clinical services, physician office visits, procedures, lab services, psychiatric services, and residential services), inpatient file (for information on nine possible diagnoses, hospitalizations, procedures, and length of stays), and the prescription drug file (for national drug codes [NDCs], prescription fill date, and days of supply). County-level variables were obtained from the area resource file $(A R F)$ [57], which provides information on type of health facilities, number and type of health professions, resource scarcity measures, health status, economic activities, health training programs, and socioeconomic and environmental characteristics. The 
Medicaid files were linked with ARF files with a five-digit county identification variable.

\subsection{Study Population and Design}

A retrospective cross-sectional matched cohort study among adults aged 22-64 years with and without ASD was conducted. Adults with any ASD (at least one inpatient or two outpatient claims on separate service dates with an ICD9-CM code: 299.xx in any position) who were continuously enrolled in a fee-for-service program from 2000 to 2008 were matched (1:3) to adults without ASD by age, gender, and race. Adults without ASD were identified as those enrollees with at least two medical claims on separate dates of service (either inpatient or outpatient) with no ASD diagnosis throughout the study period (2000-2008). Validating ASD diagnoses was outside the scope of the current study; therefore, we used a validated identification algorithm by Burke et al. [58] with a positive predictive value of $87 \%$ among children with ASD. Administrative health databases for studies concerning healthcare services utilization with validated ASD diagnoses are used more frequently now than ever [59].

Matching was conducted using propensity scores with an 8 to 1 GREEDY algorithm which involves matching cases and controls with the same propensity score (predicted probabilities from a logistic regression on ASD status) till the eighth digit, and if the eighth digit match is unsuccessful, the algorithm attempts to match on seven digits, and so on. The GREEDY matching algorithm employs a sample without replacement and if there is more than one match then selection of control becomes random. Such an approach for propensity score matching is used to reduce the effects of bias and confounding in observational studies [60]. Individuals were excluded from the study if they were (1) dual Medicaid/Medicare eligible; (2) managed-care enrollees; and (3) dead during the study period. Every year unique cases of ASD were identified and matched with a unique control from the same year with no ASD. Matched cases and controls from each year were added to make the final sample of years 2000-2008. A cohort attrition chart is provided in Fig. 1.

\subsection{Variables}

\subsubsection{Socio-Demographic Characteristics}

Age (22-40 and 41-64 years), sex (male and female), race (White, African American, and Other [Hispanics, American Indian/Alaska Native, Asian, Native Hawaiian/Other Pacific Islander, and more than one race]), state (IL, NY, and TX), identification cohort (before and after 2004), and Medicaid eligibility by cash and medical needs (yes/no) were extracted from the MAX personal summary file. These patient characteristics were used as control variables in the study.

\subsubsection{County Characteristics}

We used various contextual variables from the ARF file such as county metro status (urban/rural), primary care shortage area (yes/no), mental-health specialist shortage area (yes/no), psychiatrist density (high/low), median household income level (quartiles: q1, q2, q3, and q4), and 'high school education and above' density which is a measure of county education status (quartiles: q1, q2, q3, and q4). These were used as proxy measures of access to services.

\subsubsection{Comorbidities}

We utilized both number (none, one, and two, three or more) and type of comorbid conditions (either one inpatient or two outpatient claims on separate service dates with a diagnosis in any position for a psychiatric or a nonpsychiatric disorder) in our study. We used the single-level clinical classification software (CCS) provided by Agency for Healthcare Research and Quality [61] to categorize diagnosis codes to clinically meaningful categories.

A psychiatric comorbidity included adjustment disorders (CCS code: 650), alcohol/substance use disorders (AUD; CCS codes: 660, 661), anxiety disorders (CCS code: 651), attention-deficit disorders and conduct behavior disorders (ADD; CCS code: 652), developmental disorders (CCS code: 654), mood disorders including depressive disorders and bipolar disorder (CCS codes: 6571, 6572), personality disorders (CCS code: 658), and schizophrenia and other psychotic disorders (CCS code: 659). A nonpsychiatric comorbidity included most common medical conditions which are linked with highest healthcare expenditures and are the leading cause of deaths in the US (http://www.cdc.gov/nchs/fastats/leading-causes-of-death. htm): cancer (CCS codes: 11-37, 39-43, 46, 47) cardiovascular disease (CCS codes: 96-108), diabetes mellitus (CCS codes: 49, 50), musculoskeletal disorders (CCS codes: 201-212), gastrointestinal disease (CCS codes: 138-151, 153-155), and respiratory disease (CCS codes: 125-128, 132-134).

A variable representing number of comorbidities was created as a sum of categorical variables $(1=$ yes, $0=$ no $)$ of 'any psychiatric disorder', 'any non-psychiatric disorder', and 'other health conditions' (epilepsy, thyroid disorders, lipid metabolism disorders, pancreatic disorders, fluid and electrolyte disorders, jaw and teeth disorders, genitourinary disorders, skin disorders, and infections). As part of another study using the same cohort, the 'other 
Fig. 1 Study attrition and flow chart with inclusion/exclusion criteria. $A S D$ autism spectrum disorders, $I L$ Illinois, $I P$ inpatient, $N Y$ New York, $O T$ outpatient, $T X$ Texas

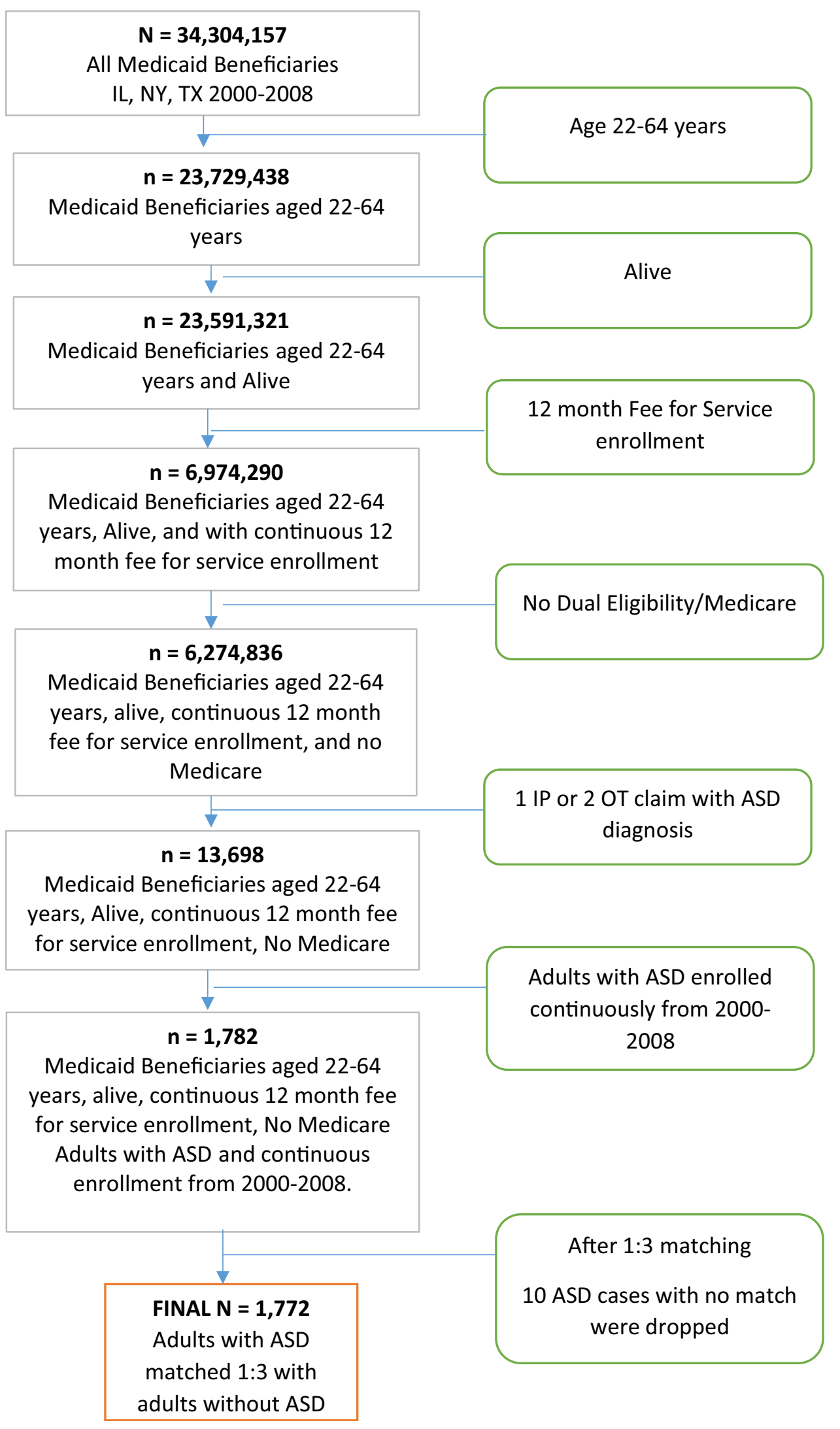


health conditions' were found to be of significant prevalence in both the ASD and no ASD group. Due to a lack of strong epidemiological evidence on comorbid prevalence of many such conditions among adults with ASD (especially in Medicaid), these were analyzed as a separate category from non-psychiatric comorbidity.

\subsubsection{Type of Prescription Drug Classes}

Overall prescription drug ( $\mathrm{Rx})$ use ( $>20$ unique Rx claims, $\leq 20$ unique Rx claims, and no Rx claims) was categorized as above or equal/below the median of the entire sample (due to non-parametric distribution of Rx use). We have reported the proportion of adults using specific drug classes as well as the mean number of prescription drug claims per year for the following drug classes: respiratory agents, antimicrobials, anticancer, immunologics, cardiovascular agents, analgesics, blood modifiers, hormone modifiers/ steroids, statins/other antihyperlipidemics, antidiabetics, dermatologic agents, alternative medicine/nutritional supplements, gastrointestinal agents, CNS agents (excluding stimulants), antiparkinsonian agents, and other metabolic agents. The list of specific drugs within these prescription drug classes is provided in Appendix A (see electronic supplementary material). These agents were identified using a drug database from Multum ${ }^{\circledR}$ Lexicon 2014 (Cerner Multum, Inc., Denver, CO, USA), which provides national drug codes or NDCs (11-digit code). Such NDCs represent unique prescription drugs that are assigned drug categories which can be classified further into meaningful therapeutic classes and can be linked with the MAX pharmacy file.

\subsubsection{Polypharmacy}

We defined general polypharmacy (yes, no, no Rx claim) as use of six or more unique classes of prescription drugs in a year. General polypharmacy included but was not restricted to prescription drug classes such as antipsychotics, antidiabetics, antihyperlipidemics, anticancer drugs, antibiotics, gastrointestinal agents, respiratory agents, and other classes of drugs (Appendix A; see electronic supplementary material). The psychotropic drug polypharmacy (yes, no, no Rx claim) was defined as use of three or more unique classes of psychotropic drugs within a 90-day period after the first occurring prescription fill date of any psychotropic drug. A similar definition of psychotropic polypharmacy in claims database analyses has been previously used by Essock et al. [47] among individuals of all ages and Spencer et al. [39] among children with ASD. The psychotropic drug classes in the current study included antidepressants, antipsychotics, anticonvulsants, CNS stimulants, and anxiolytics/sedatives/ hypnotics. For converting the national drug codes to meaningful therapeutic drug classes, we used drug database from Multum ${ }^{\circledR}$ Lexicon 2014 (Cerner Multum, Inc., Denver, CO, USA) and Healthcare Effectiveness Data and Information Set (HEDIS) NDC 2015 lists [62].

\subsection{Statistical Analyses}

Bivariate analyses such as Chi-square tests of associations for categorical variables and $t$ tests for continuous variables were conducted to assess the subgroup and mean differences between adults with and without ASD. Mean annual numbers of claims for adults with and without ASD were calculated for all individuals in the data as well as only users (those who had at least one prescription drug claim for a specific therapeutic drug class). Binary logistic regressions were run to assess the effect sizes of differences in rates of prescription drug use among adults with and without ASD (adjusted for age, sex, and race). To examine the likelihood of general and psychotropic polypharmacy, multinomial logistic regressions were run.

Regression analyses were adjusted for socio-demographics, county characteristics, and number of comorbidities. For examining characteristics of adults with ASD significantly associated with polypharmacy, the multinomial variable was converted to a binary indicator (polypharmacy vs no polypharmacy). Chi square analyses and binary logistic regressions were conducted to identify significant factors of adults with ASD associated with polypharmacy. Numbers, percentages, means and standard deviations, adjusted odds ratios (AORs) along with 95\% confidence intervals (CIs) are reported. Multinomial logistic regression models were controlled for cohort (before/after 2004), state, eligibility, county characteristics (metro status, median household income, above high school education, primary care provider shortage area, mental health specialist shortage area, and psychiatrist density), and number of comorbidities. Findings with cell sizes that were 11 or less are suppressed in accordance with CMS data user agreement. All analyses were conducted using SAS v9.4.

\section{Results}

Our study sample had the maximum number of enrollees in the year 2000 ( $n=2468,34.8 \%)$ and the minimum number of enrollees in the year $2007(n=696,9.8 \%)$. It consisted of adults who were primarily male $(n=5068$, $71.5 \%)$, aged $22-40$ years $(n=6072,85.6 \%)$ and belonged to the 'Other' race $(n=3002,42.3 \%)$. The majority of adults resided in a metro region $(n=6020,88 \%)$, in a physician care shortage area $(n=6140,90 \%)$, and in a 
mental health specialist shortage area $(n=5608,82 \%)$, had a high median annual household income (q4; $n=5496,80 \%)$ and were well educated (q4; $n=4468$, $65 \%)$. The sample had 1772 adults with ASD, most of them being male (71\%), aged 22-40 years, and under a cash assistance program $(82 \%)$. Rates of psychiatric $(81 \%$ ASD vs $\left.41 \%_{\text {noASD }}\right)$ and non-psychiatric comorbidity $\left(40 \%_{\text {ASD }}\right.$ vs $45 \%_{\text {noASD }}$ ) were significantly different for adults with and without ASD. Around 4\% of adults with ASD were Medicaid eligible by medical needs compared with $17 \%$ of adults without ASD. About $82 \%$ of adults with ASD were Medicaid eligible by cash assistance compared with $60 \%$ of adults without ASD.

\subsection{Types of Prescription Drug Classes}

Almost $95 \%$ of adults with ASD had at least one prescription drug claim per year compared with $87 \%$ of adults without ASD. A significantly greater proportion of adults with ASD used dermatologic agents $\left(48 \%_{\text {ASD }}\right.$ vs $\left.32 \%_{\text {noASD }}\right)$, gastrointestinal agents $\left(32 \%_{\text {ASD }}\right.$ vs $\left.23 \%_{\text {noASD }}\right)$, alternative medicine/nutritional supplements $(25 \%$ ASD vs $\left.18 \%_{\text {noASD }}\right)$, cardiovascular agents $\left(23 \%_{\text {ASD }}\right.$ vs $\left.19 \%_{\text {noASD }}\right)$, antiparkinsonian agents $\left(23 \%_{\text {ASD }}\right.$ vs $\left.10 \%_{\text {noASD }}\right)$, statins/ other antihyperlipidemics $\left(7.3 \%_{\mathrm{ASD}}\right.$ vs $\left.5 \%_{\text {noASD }}\right)$, immunologics $\left(2 \%_{\mathrm{ASD}}\right.$ vs $\left.0.7 \%_{\text {noASD }}\right)$, and other metabolic agents $\left(1.4 \%_{\text {ASD }}\right.$ vs $\left.1.1 \%_{\text {noASD }}\right)$. Psychotropic drug use was also significantly higher among adults with ASD (85\%) compared with adults without ASD (42\%). Within psychotropic drug classes, antidepressants $\left(37 \%_{\text {ASD }}\right.$ vs $\left.26 \%_{\text {noASD }}\right)$, antipsychotics $\left(66 \%_{\text {ASD }}\right.$ vs $\left.20 \%_{\text {noASD }}\right)$, anticonvulsants $\left(59 \%_{\text {ASD }}\right.$ vs $20 \%$ noASD $)$, and anxiolytics/hypnotics/sedatives $\left(21 \%_{\text {ASD }}\right.$ vs $\left.11 \%_{\text {noASD }}\right)$ were very commonly used by adults with ASD (Table 1).

\subsection{Number of Prescription Drug Claims}

On average, adults with ASD had a significantly higher number of prescription drug claims per year $\left(48_{\mathrm{ASD}}\right.$ vs $21_{\text {noASD }}$ claims). The difference in mean number of psychotropic drug claims between adults with and without ASD was highest for anticonvulsants $\left(10_{\mathrm{ASD}}\right.$ vs $2_{\text {noASD }}$ claims), followed by antipsychotics $\left(10_{\mathrm{ASD}}\right.$ vs $2_{\text {noASD }}$ claims) and antidepressants (4ASD vs $2_{\text {noASD }}$ claims) (Tables 2, 3).

\subsection{Polypharmacy among Adults with and Without ASD}

The rates of general polypharmacy among adults with ASD and without ASD were $48 \%$ and $32 \%$, respectively (AOR $1.17,95 \%$ CI 1.03-1.33). Psychotropic polypharmacy was prevalent among $19 \%$ of adults with ASD compared with
$6 \%$ of adults without ASD (AOR 1.40, 95\% CI 1.17-1.68). Most common prescription drug classes used by these adults were antipsychotics (78\%), anticonvulsants (75\%), antimicrobials (67\%), dermatologic agents (64\%), respiratory agents (59\%), and gastrointestinal agents (52\%) (Table 1).

\subsection{Characteristics of Adults with ASD Associated with General Polypharmacy}

General polypharmacy rates increased significantly and consistently from 2000 (42\%) to 2007 (64\%). A significantly greater proportion of females with ASD (55\%) used six or more drug classes per year compared with males with ASD (45\%). Higher general polypharmacy rates for adults with ASD were observed in the 41- to 64-year age group (61\%) compared with the 22- to 40-year age group, and in Whites (54\%) compared with African Americans (47\%) and 'Other' races (44\%). Around $52 \%$ of adults with ASD and a psychiatric comorbidity used six or more drug classes in a year compared with $32 \%$ of adults with ASD but no psychiatric comorbidity. The highest rates of general polypharmacy among adults with ASD were seen for those who had personality disorders (80\%), schizophrenia (66\%), mood disorders (65\%), a gastrointestinal disorder (78\%), cardiovascular/cerebrovascular disorders $(74 \%)$, a respiratory disorder $(71 \%)$, musculoskeletal disorders $(68 \%)$, and diabetes (64\%). Around $21 \%$ of adults with ASD with no comorbidity used six or more drug classes per year. Among adults with ASD, significant predictors of general polypharmacy were males compared with females (AOR $0.63,95 \%$ CI $0.50-0.80$ ) and residence in a county with lower versus higher education status (q1 vs q4; AOR 0.43 , 95\% CI 0.27-0.66) (Table 4).

\subsection{Characteristics of Adults with ASD Associated with Psychotropic Polypharmacy}

White race had a significantly greater psychotropic polypharmacy rate compared with African Americans and 'Other' races. Adults with ASD and a psychiatric comorbidity such as an adjustment disorder (26\%), mood disorder $(31 \%)$, or schizophrenia (32\%) had significantly high rates of psychotropic polypharmacy. Around $12 \%$ of adults with ASD with no comorbidity used psychotropic polypharmacy. In the multivariate regression analyses, adults with ASD who were either African American (AOR 0.50, 95\% CI 0.34-0.72) or 'Other' race (AOR 0.71, 95\% CI 0.53-0.96) had lower odds of psychotropic polypharmacy compared with Whites.

Greater numbers of comorbidities were associated with greater general and psychotropic polypharmacy rates 
Table 1 Description of type of prescription drugs and polypharmacy by ASD status. Adults with and without ASD matched on age, race, and gender $(n=7092)$. Medicaid Analytic Extract 2000-2008 (IL, NY, and TX)

\begin{tabular}{|c|c|c|c|c|c|c|}
\hline \multirow{2}{*}{ Rx type } & \multicolumn{2}{|l|}{ ASD } & \multicolumn{2}{|c|}{ No ASD } & \multirow{2}{*}{$\begin{array}{l}\text { AOR } \\
\text { ASD vs. no ASD }\end{array}$} & \multirow[t]{2}{*}{$95 \% \mathrm{CI}$} \\
\hline & $N$ & $\mathrm{Col} \%$ & $N$ & $\mathrm{Col} \%$ & & \\
\hline \multicolumn{7}{|l|}{ Any prescription drug ${ }^{b}$} \\
\hline$>20 \mathrm{Rx}$ claims & 1,325 & 74.7 & 1,766 & 33.2 & & \\
\hline$\leq 20 \mathrm{Rx}$ claims & 353 & 19.9 & 2,857 & 53.7 & & \\
\hline no claim & 94 & 5.3 & 697 & 13.1 & & \\
\hline Dermatologic agents & 850 & 48.0 & 1,711 & 32.2 & $1.75^{* * *}$ & $(1.57,1.96)$ \\
\hline Respiratory agents & 679 & 38.3 & 1,821 & 34.2 & 1.05 & $(0.93,1.17)$ \\
\hline Gastrointestinal agents & 560 & 31.6 & 1,235 & 23.2 & $1.39 * * *$ & $(1.23,1.57)$ \\
\hline Miscellaneous agents & 514 & 29.0 & 924 & 17.4 & $1.79 * * *$ & $(1.57,2.03)$ \\
\hline Alternative medicine/nutritional supplements & 437 & 24.7 & 964 & 18.1 & $1.35 * * *$ & $(1.19,1.55)$ \\
\hline Cardiovascular agents & 411 & 23.2 & 1,026 & 19.3 & $1.14^{*}$ & $(1.02,1.31)$ \\
\hline Antiparkinson agents & 401 & 22.6 & 523 & 9.8 & $2.50 * * *$ & $(2.16,2.89)$ \\
\hline Statins/other antihyperlipidemics & 129 & 7.3 & 266 & 5.0 & $1.39 * *$ & $(1.11,1.73)$ \\
\hline Immunologics & 35 & 2.0 & 37 & 0.7 & $2.65 * * *$ & $(1.66,4.23)$ \\
\hline Other metabolic agents & 25 & 1.4 & 57 & 1.1 & 1.21 & $(0.75,1.95)$ \\
\hline Antimicrobials & 822 & 46.4 & 2,913 & 54.8 & $0.56 * * *$ & $(0.50,0.63)$ \\
\hline Analgesics & 503 & 28.4 & 2,615 & 49.2 & $0.32 * * *$ & $(0.29,0.36)$ \\
\hline Hormone modifiers/steroids & 281 & 15.9 & 909 & 17.1 & $0.81 * *$ & $(0.69,0.94)$ \\
\hline CNS agents (excluding stimulants) & 74 & 4.2 & 540 & 10.2 & $0.35 * * *$ & $(0.27,0.45)$ \\
\hline Antidiabetics & 62 & 3.5 & 262 & 4.9 & $0.64 * *$ & $(0.48,0.85)$ \\
\hline Blood modifiers & 44 & 2.5 & 219 & 4.1 & $0.53 * * *$ & $(0.38,0.74)$ \\
\hline Anticancer $^{\mathrm{a}}$ & 13 & 0.7 & 121 & 2.3 & & \\
\hline Psychotropic medications & 1,510 & 85.2 & 2,254 & 42.4 & $9.79 * * *$ & $(8.25,11.6)$ \\
\hline Antipsychotics & 1,170 & 66.0 & 1,072 & 20.2 & $7.97 * * *$ & $(7.02,9.05)$ \\
\hline Anticonvulsants & 1,048 & 59.1 & 1,042 & 19.6 & $5.85 * * *$ & $(5.18,6.61)$ \\
\hline Antidepressants & 652 & 36.8 & 1,361 & 25.6 & $1.54 * * *$ & $(1.36,1.73)$ \\
\hline Anxiolytics/Hypnotics/Sedatives & 380 & 21.4 & 587 & 11.0 & $2.03 * * *$ & $(1.76,2.34)$ \\
\hline CNS stimulants & 33 & 1.9 & 54 & 1.0 & $1.69 *$ & $(1.09,2.62)$ \\
\hline Polypharmacy & & & & & & \\
\hline General polypharmacy ( $\geq 6$ classes) & 856 & 48.3 & 1,727 & 32.5 & & \\
\hline Psychotropic polypharmacy ( $\geq 3$ classes) & 337 & 19.0 & 312 & 5.9 & & \\
\hline ASD vs. no ASD & OR $(95$ & CI ) & AOR $(9$ & CI) & & \\
\hline$\geq 6$ classes vs. $<6$ classes & $1.74 * * *$ & $(1.56,1.95)$ & $1.17 *$ & $(1.03,1.33)$ & & \\
\hline$\geq 3$ vs. $<3$ psychotropic classes & $1.65 * * *$ & $(1.39,1.96)$ & $1.40 * * *$ & $(1.17,1.68)$ & & \\
\hline
\end{tabular}

Based on chi-square estimates for fee for service enrolled individuals aged 22-64 years with no Medicare coverage and alive in IL, NY, and TX Medicaid from 2000-2008. Fischer exact tests were conducted for variables with expected cell count $<5$

ASD Autism Spectrum Disorders, Rx Prescription Drug, Col \% Column percentages, OR Odds ratio, AOR Adjusted odds ratio

$* * * p<0.001 ; * * 0.001 \leq p<0.01 ; * 0.01 \leq p<0.05$

${ }^{\text {a }}$ Chi-square test was not conducted due to very low cell sizes. All chi-square associations were significant at $* * * \mathrm{P}<0.001$

b Estimates also tested with Wilcoxon-Mann Whitney tests

among adults with ASD. Having three or more comorbidities among adults with ASD was strongly associated with greater odds of general (AOR 11.14, 95\% CI 7.05-17.6) and psychotropic polypharmacy (AOR 3.03, 95\% CI 1.76-5.22) (Table 4).

\section{Discussion}

The use of prescription drugs among Medicaid-enrolled adults with ASD is substantial and is significantly greater than in an age-, gender-, race-matched sample of adults 
Table 2 Mean number of claims for specific Rx classes per year. Adults with and without ASD matched on age, race, and gender $(\mathrm{n}=7092)$ Medicaid Analytic Extract 2000-2008 (IL, NY, and TX)

\begin{tabular}{|c|c|c|c|c|}
\hline Rx type & $\begin{array}{l}\text { ASD } \\
\text { Mean (SD) }\end{array}$ & $\begin{array}{l}\text { No ASD } \\
\text { Mean (SD) }\end{array}$ & $\begin{array}{l}\text { Ratio of Means } \\
\text { ASD vs. No ASD }\end{array}$ & Sig. \\
\hline Any prescription drug & $48.15(37)$ & $21.36(29)$ & 2.25 & $* * *$ \\
\hline Respiratory agents & $2.50(6)$ & $1.85(6)$ & 1.35 & $* *$ \\
\hline Antimicrobials & $1.34(3)$ & $2.79(8)$ & 0.48 & $* * *$ \\
\hline Anticancer & $0.07(2)$ & $0.03(1)$ & 0.00 & \\
\hline Immunologics & $0.02(0)$ & $0.03(1)$ & 0.00 & \\
\hline Cardiovascular agents & $2.77(7)$ & $1.77(6)$ & 1.56 & $* * *$ \\
\hline Analgesics & $0.85(2)$ & $2.11(5)$ & 0.40 & $* * *$ \\
\hline Blood modifiers & $0.20(1)$ & $0.23(2)$ & 0.00 & \\
\hline Hormone modifiers/steroids & $1.44(4)$ & $0.78(3)$ & 1.85 & $* * *$ \\
\hline Statins/other antihyperlipidemics & $0.74(3)$ & $0.27(2)$ & 2.74 & $* * *$ \\
\hline Antidiabetics & $0.40(3)$ & $0.50(3)$ & 0.80 & \\
\hline Dermatologic agents & $2.83(6)$ & $1.16(3)$ & 2.44 & $* * *$ \\
\hline Alternative medicine/nutritional supplements & $2.38(6)$ & $0.87(3)$ & 2.74 & $* * *$ \\
\hline Gastrointestinal agents & $3.52(8)$ & $1.16(4)$ & 3.03 & $* * *$ \\
\hline CNS agents (excluding stimulants) & $0.37(2)$ & $0.35(2)$ & 1.00 & \\
\hline Antiparkinsonian agents & $1.86(4)$ & $0.48(2)$ & 3.88 & $* * *$ \\
\hline Other metabolic agents & $0.15(1)$ & $0.05(1)$ & 3.00 & $* * *$ \\
\hline Psychotropic medications & $25.51(21)$ & $6.26(12)$ & 4.08 & $* * *$ \\
\hline Antidepressants & $3.93(6)$ & $1.79(4)$ & 2.20 & $* * *$ \\
\hline Antipsychotics & $9.90(10)$ & $2.04(6)$ & 4.85 & $* * *$ \\
\hline Anticonvulsants & $10.28(14)$ & $1.84(6)$ & 5.59 & $* * *$ \\
\hline CNS stimulants & $0.14(1)$ & $0.05(1)$ & 2.80 & $* * *$ \\
\hline Anxiolytics/Hypnotics/Sedatives & $1.25(3)$ & $0.54(2)$ & 2.31 & $* * *$ \\
\hline
\end{tabular}

Based on t-test estimates for fee for service enrolled individuals aged 22-64 years with no Medicare coverage and alive in IL, NY, and TX Medicaid from 2000-2008

$* * * p<0.001 ; * * 0.001 \leq p<0.01 ; * 0.01 \leq p<0.05$ without ASD. The higher medication burden is only observed across a high number of adults with ASD, but also extends to a high annual mean number of prescription fills. Adults with ASD not only use a considerable amount of prescriptions drugs for psychiatric needs such as antipsychotics, antidepressants, and anxiolytics/sedatives, but also use prescription drugs that alleviate medical issues including gastrointestinal agents, cardiovascular agents, statins, dermatologics, and many others. Another notable finding of the current study was the rates of polypharmacy among adults with ASD. Almost half of the adults with ASD used six or more drug classes in a year and $19 \%$ of adults with ASD used three or more psychotropic drug classes within a 90-day period. Among adults with ASD but no comorbidity, about $20 \%$ used six or more prescription drug classes in a year and $12 \%$ used three or more psychotropic drugs in a 90-day period, indicating a possible off-label prescription drug use.

With the rising concerns of off-label use and potential risks associated with multiple drug use among individuals with ASD [10], our study provides critical information on prescription practices and the patterns of drug utilization among adults with ASD. Various studies in the past have focused on examining the extent of medication use and polypharmacy among children and adolescents with ASD $[12,22,25,26,39,40,55,63]$, but the current study is the first large sample study that has established rates of prescription drug use, types of prescription drug classes used, and types of polypharmacy among adults with ASD compared with adults without ASD.

\subsection{Overall and Specific Prescription Drug Use}

A large majority of adults with ASD (85\%) filled at least one prescription for a psychotropic drug, which was significantly higher than adults without ASD (42\%). Compared with earlier studies on psychotropic medication and polypharmacy among adults with ASD [19, 21], psychotropic drug use rates in the current study were much higher than the previous rates of 64\% (19) and 57\% [21]. Unlike the study by Esbensen et al. [21], which focused on a younger age group of 10- to 48-year-old individuals with 
Table 3 Mean number of claims for specific Rx classes per year for users ${ }^{\mathrm{a}}$. Adults with and without ASD matched on age, race, and gender $(\mathrm{n}=$ 7092). Medicaid Analytic Extract 2000-2008 (IL, NY, and TX)

\begin{tabular}{|c|c|c|c|c|}
\hline & $\begin{array}{l}\text { ASD } \\
\text { Mean (SD) }\end{array}$ & $\begin{array}{l}\text { No ASD } \\
\text { Mean (SD) }\end{array}$ & $\begin{array}{l}\text { Ratio of Means } \\
\text { ASD vs. No } \\
\text { ASD }\end{array}$ & Sig. \\
\hline Any prescription drug & $50(36)$ & $25(30)$ & 2.00 & $* * *$ \\
\hline Respiratory agents & $7(8)$ & $5(8)$ & 1.40 & $* *$ \\
\hline Antimicrobials & $3(3)$ & $5(10)$ & 0.60 & $* * *$ \\
\hline Anticancer & $10(17)$ & $6(7)$ & 1.66 & \\
\hline Immunologics & $1(0)$ & $4(6)$ & 0.25 & $* * *$ \\
\hline Cardiovascular agents & $12(9)$ & $9(10)$ & 1.33 & $* * *$ \\
\hline Analgesics & $3(3)$ & $4(6)$ & 0.75 & $* * *$ \\
\hline Blood modifiers & $8(6)$ & $6(6)$ & 1.33 & \\
\hline Hormone modifiers/steroids & $9(6)$ & $5(5)$ & 1.80 & $* * *$ \\
\hline Statins/other antihyperlipidemics & $10(5)$ & $5(4)$ & 2.00 & $* * *$ \\
\hline Antidiabetics & $12(8)$ & $10(9)$ & 1.20 & \\
\hline Dermatologic agents & $6(7)$ & $4(5)$ & 1.50 & $* * *$ \\
\hline Alternative medicine/nutritional supplements & $10(8)$ & $5(6)$ & 2.00 & $* * *$ \\
\hline Gastrointestinal agents & $11(10)$ & $5(6)$ & 2.20 & $* * *$ \\
\hline CNS agents (excluding stimulants) & $9(7)$ & $3(4)$ & 3.00 & $* * *$ \\
\hline Antiparkinsonian agents & $8(6)$ & $5(5)$ & 1.60 & $* * *$ \\
\hline Other metabolic agents & $10(5)$ & $5(4)$ & 2.00 & $* * *$ \\
\hline Psychotropic medications & $30(20)$ & $15(16)$ & 2.00 & $* * *$ \\
\hline Antidepressants & $11(6)$ & $7(6)$ & 1.57 & $* * *$ \\
\hline Antipsychotics & $15(9)$ & $10(10)$ & 1.50 & $* * *$ \\
\hline Anticonvulsants & $17(14)$ & $9(9)$ & 1.88 & $* * *$ \\
\hline CNS stimulants & $7(6)$ & $5(4)$ & 1.40 & $* *$ \\
\hline Anxiolytics/Hypnotics/Sedatives & $6(5)$ & $5(5)$ & 1.20 & $* *$ \\
\hline
\end{tabular}

Based on t-test estimates for fee for service enrolled individuals aged 22-64 years with no Medicare coverage and alive in IL, NY, and TX Medicaid from 2000-2008

$* * * p<0.001 ; * * 0.001 \leq p<0.01 ; * 0.01 \leq p<0.05$

a Only among those who used specific drug classes
ASD, our study included adults up to 64 years of age, which could have been the reason for elevated rates of prescription drug use. The study by Lake et al. [19], although very informative, was restricted by the clinical settings, staff reports of medication use, and a low sample size which may not be representative of communitydwelling adults with ASD. Nonetheless, the rate of psychotropic drug use in our study was much higher than the rates among children with ASD (27-64\%), as observed in previous studies with and without a Medicaid database [39, 41, 63].

A high proportion of adults with ASD in the current study also used non-psychotropic drugs, indicating the nonpsychiatric needs of this population. Previous studies have mainly focused on psychiatric needs and utilization of psychotropic drugs among individuals with ASD $[12,22,25,26,39,40,55,63]$, and our study is the first one to examine the rates of non-psychotropics such as dermatologic agents (48\%), gastrointestinal agents (32\%), alternative medicine/nutritional supplements (25\%), cardiovascular agents (23\%), antiparkinsonian agents (23\%), and statins/other antihyperlipidemics (7.3\%) among adults with ASD. Our study results support the findings of recent observational studies that have highlighted the nonpsychiatric needs of individuals with ASD (55). Croen et al. [54], using claims data from the Northern California Kaiser Permanente program, showed a considerable and higher prevalence of non-psychiatric comorbidities such as immune conditions, gastrointestinal disorders, dyslipidemia, hypertension, and diabetes among adults with ASD compared with adults without ASD. In fact, adults with ASD also had a higher prevalence of rare disorders such as stroke and Parkinsonism. Recently, a study by Starkstein et al. [64] showed a $20 \%$ prevalence of Parkinsonism among a small sample of middle aged and older adults with ASD (49 years and above). Hollander et al. [65] published a review on links between Parkinsonism and ASD which may have overlapping phenomenology such as repetitive behaviors and a common underlying involvement of the basal ganglia leading to symptoms that require similar 
Table 4 Description of Sample by General and Psychotropic Polypharmacy Among Adults with ASD ( $\mathrm{n}=1772)$. Medicaid Analytic Extract 2000-2008 (IL, NY, and TX)

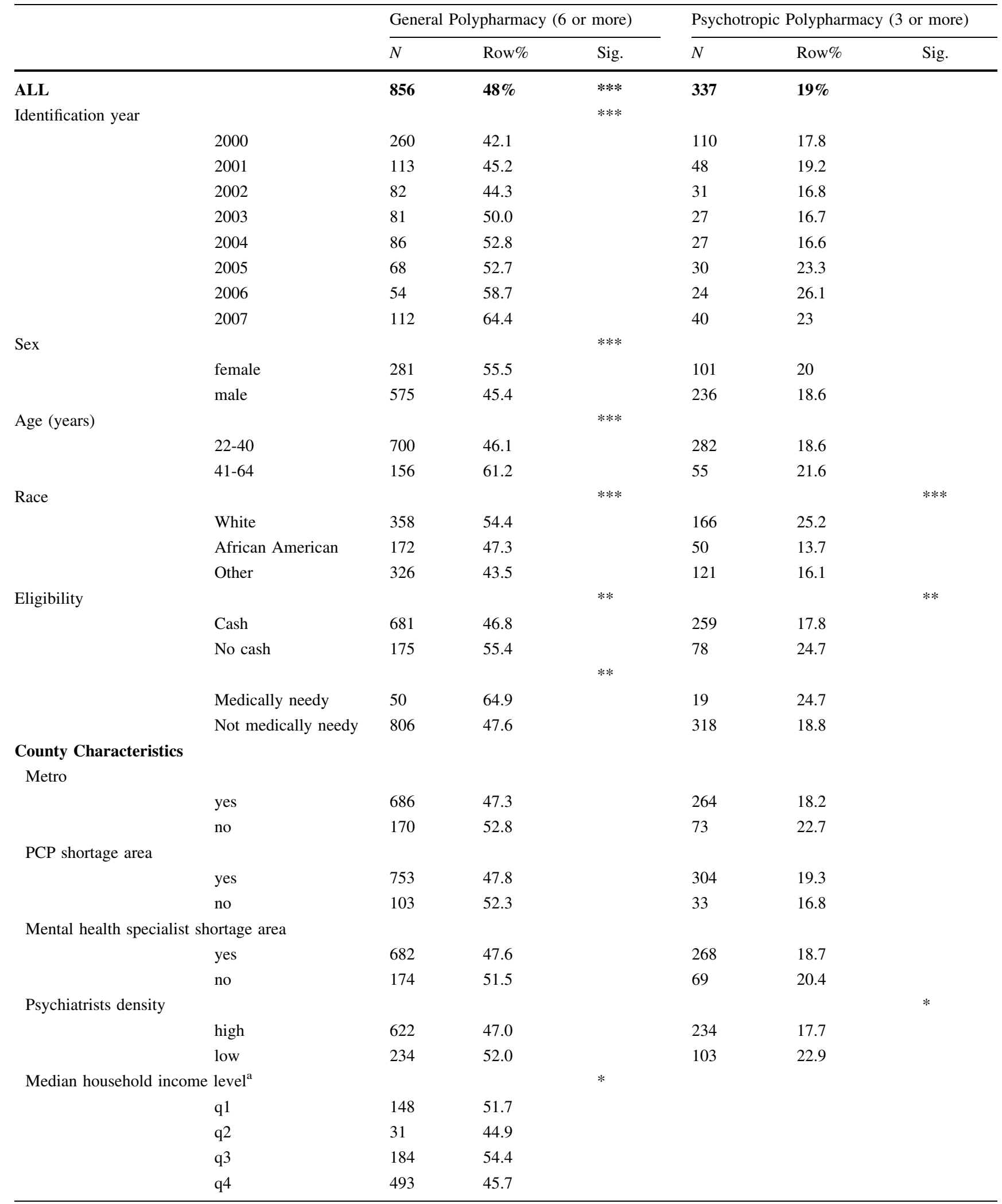


Table 4 continued

\begin{tabular}{|c|c|c|c|c|c|c|c|}
\hline & & \multicolumn{3}{|c|}{ General Polypharmacy (6 or more) } & \multicolumn{3}{|c|}{ Psychotropic Polypharmacy ( 3 or more) } \\
\hline & & $N$ & Row\% & Sig. & $N$ & Row\% & Sig. \\
\hline \multirow[t]{5}{*}{ HS education and above ${ }^{a}$} & & & & $*$ & & & \\
\hline & $\mathrm{q} 1$ & 494 & 46.1 & & & & \\
\hline & $\mathrm{q} 2$ & 34 & 42.5 & & & & \\
\hline & $\mathrm{q} 3$ & 130 & 53.3 & & & & \\
\hline & $\mathrm{q} 4$ & 198 & 52.5 & & & & \\
\hline \multicolumn{8}{|l|}{ Comorbidities } \\
\hline \multirow[t]{3}{*}{ Psychiatric Comorbidity } & & & & $* * *$ & & & $*$ \\
\hline & yes & 750 & 52.1 & & 289 & 20.1 & \\
\hline & no & 106 & 31.9 & & 48 & 14.5 & \\
\hline \multicolumn{8}{|l|}{ Adjustment Disorders } \\
\hline & yes & 26 & 44.8 & & 15 & 25.9 & \\
\hline & no & 830 & 48.4 & & 322 & 18.8 & \\
\hline \multirow[t]{3}{*}{ Anxiety } & & & & & & & $*$ \\
\hline & yes & 117 & 54.2 & & 53 & 24.5 & \\
\hline & no & 739 & 47.5 & & 284 & 18.3 & \\
\hline \multirow[t]{3}{*}{ ADD/ADHD } & & & & $*$ & & & $* * *$ \\
\hline & yes & 82 & 56.2 & & 43 & 29.5 & \\
\hline & no & 774 & 47.6 & & 294 & 18.1 & \\
\hline \multirow[t]{3}{*}{ Intellectual Disabilities } & & & & $*$ & & & \\
\hline & yes & 627 & 50.9 & & 224 & 18.2 & \\
\hline & no & 229 & 42.3 & & 113 & 20.9 & \\
\hline \multirow[t]{3}{*}{ Mood Disorders } & & & & $* * *$ & & & $* * *$ \\
\hline & yes & 165 & 64.5 & & 79 & 30.9 & \\
\hline & no & 691 & 45.6 & & 258 & 17 & \\
\hline \multirow[t]{3}{*}{ Schizophrenia } & & & & $* * *$ & & & $* * *$ \\
\hline & yes & 194 & 66.0 & & 20 & 45.5 & \\
\hline & no & 662 & 44.8 & & 317 & 18.3 & \\
\hline \multicolumn{2}{|l|}{ Non-Psychiatric Comorbidity } & & & $* * *$ & & & \\
\hline & yes & 481 & 67.9 & & 137 & 19.4 & \\
\hline & no & 375 & 35.2 & & 200 & 18.8 & \\
\hline \multicolumn{2}{|l|}{ Gastrointestinal Disorders } & & & $* * *$ & & & \\
\hline & yes & 173 & 77.6 & & 48 & 21.5 & \\
\hline & no & 683 & 44.1 & & 289 & 18.7 & \\
\hline \multirow[t]{3}{*}{ Respiratory Disorders } & & & & $* * *$ & & & \\
\hline & yes & 190 & 71.2 & & 57 & 21.3 & \\
\hline & no & 666 & 44.3 & & 280 & 18.6 & \\
\hline \multirow[t]{3}{*}{ Muscoskeletal Disorders } & & & & $* * *$ & & & \\
\hline & yes & 147 & 68.1 & & 35 & 16.2 & \\
\hline & no & 709 & 45.6 & & 302 & 19.4 & \\
\hline \multicolumn{2}{|c|}{ Cardiovascular/Cerebrovascular Disorders } & & & $* * *$ & & & \\
\hline & yes & 179 & 74.0 & & 50 & 20.7 & \\
\hline & no & 677 & 44.2 & & 287 & 18.8 & \\
\hline \multirow[t]{3}{*}{ Diabetes $^{\mathrm{a}}$} & & & & $*$ & & & \\
\hline & yes & 40 & 63.5 & & - & & \\
\hline & no & 816 & 47.7 & & & & \\
\hline
\end{tabular}


Table 4 continued

\begin{tabular}{|c|c|c|c|c|c|c|c|}
\hline & & \multicolumn{3}{|c|}{ General Polypharmacy ( 6 or more) } & \multicolumn{3}{|c|}{ Psychotropic Polypharmacy ( 3 or more) } \\
\hline & & $N$ & Row\% & Sig. & $N$ & Row\% & Sig. \\
\hline \multirow[t]{3}{*}{ Epilepsy } & & & & $* * *$ & & & $* *$ \\
\hline & yes & 255 & 63.3 & & 98 & 24.3 & \\
\hline & no & 601 & 43.9 & & 239 & 17.5 & \\
\hline \multirow[t]{5}{*}{ Number of comorbidities } & & & & $* * *$ & & & $* * *$ \\
\hline & None & 33 & 20.6 & & 19 & 11.9 & \\
\hline & 1 & 101 & 27.3 & & 53 & 14.3 & \\
\hline & 2 & 151 & 41.3 & & 61 & 16.7 & \\
\hline & 3 or more & 571 & 65.2 & & 204 & 23.3 & \\
\hline
\end{tabular}

Based on chi-square estimates for fee for service enrolled individuals aged 22-64 years with no Medicare coverage and alive in IL, NY, and TX Medicaid from 2000-2008. Fischer exact tests were conducted for variables with expected cell count $<5$

Polypharmacy variable was a three category variable (yes, no, no Rx claim). Numbers and \% shown here represent the first category only ASD Autism Spectrum Disorders, Rx Prescription Drug, PCP Primary Care Provider, HS High School

$* * * p<0.001 ; * * 0.001 \leq p<0.01 ; * 0.01 \leq p<0.05$

${ }^{a}$ Chi-square tests were not conducted and findings are not presented for cell sizes $\leq 11$

medication management. Use of additional prescription drugs other than psychotropic drugs may be of bigger concern in a scenario where numerous medications are used off-label with no information on their benefit-risk ratios, limited data on beneficial effects of psychotropic drugs other than risperidone and aripiprazole [15], and a lack of well designed clinical trials with large cohorts of the adult ASD population [10].

In the current study, adults with ASD also had a significantly greater mean number of prescription drug claims per year than adults without ASD. For some drug classes such as anticonvulsants, the mean number of claims per year was as high as 11 claims per year for adults with ASD compared with a mean of two claims per year for adults without ASD. These findings were consistent when restricted to users only, where adults with ASD still had a higher mean number of claims in a year for most drug classes. In a 2006 study by Croen et al., Medicaid-enrolled children with ASD had an average of six prescription drug claims per year [66] compared with our study rate of 48 prescription drug claims per year among adults with ASD. Findings of the study by Esbensen et al. [21], which found that young adults are less likely to discontinue medications and more likely to add medications over the course of the ASD treatment, strengthen the hypothesis that adults with ASD may have much higher prescription needs than children with ASD.

\subsection{General Polypharmacy}

In our study cohort, almost half of adults with ASD used six or more prescription drug classes in a year compared with one-third of adults without ASD. One of the interesting findings in our study was the consistent increase ( $22 \%$ rise) in the proportion of adults with ASD who engaged in general polypharmacy from 2000 to 2007. The past decade was a critical transition period for ASD awareness and better diagnoses [67], better Medicaid policies for prescription drug coverage for individuals with mental health issues such as ASD [48, 68], and an increased mental health workforce that could have been associated with better access to medications leading to an increased number of individuals getting easier access to prescription drugs. However, this finding should be read with caution because our study could not establish causality of rising polypharmacy rates with time. Adults with ASD living in counties with workforce shortages of primary care and mental health specialty providers had lower rates of general polypharmacy than those living in counties with no workforce shortage, underlining the important role of healthcare providers in access to prescription drugs for adults with ASD and emphasizing the need for better pharmacological guidelines to help providers to care for adults with ASD, and reduce excess prescription drug use whenever risks outweigh benefits.

Among predictors of general polypharmacy for adults with ASD, female gender and older age was significantly associated with using six or more drug classes per year compared with male gender and younger age group, respectively. Albeit not fully established, a few studies have shown that females with ASD are less likely to have repetitive behaviors but have a higher prevalence of other disorders such as intellectual disabilities, anxiety, schizophrenia, depression, bipolar disorders, cancer, 
diabetes, and some neurological disorders, putting them at a greater risk of using multiple classes of drugs [54, 69].

\subsection{Psychotropic Polypharmacy}

Psychotropic polypharmacy was observed among 19\% of adults with ASD compared with only $6 \%$ of adults without ASD. This rate of psychotropic polypharmacy was lower than a rate of $29 \%$ reported by Lake et al. (2012) among adults with ASD [19] and higher than the $20 \%$ rate among children with ASD [39, 41]. Adults with ASD who had adjustment disorders (26\%), ADD/ADHD (30\%), mood disorders (31\%), schizophrenia (32\%), and epilepsy (24\%) had greater psychotropic polypharmacy rates than adults with ASD but no respective comorbidities. Around $12 \%$ of adults with ASD (Table 4) but no comorbidity used three or more psychotropic drugs in a 90-day period. This finding reflects that physicians are possibly following off-label prescription practices without strong evidence for drugs such as SSRIs and drugs such as memantine for improvements in autistic symptoms such as irritability, hyperactivity, and aggression [54, 69]. In addition, use of multiple classes of drugs at one time also increases the risks of adverse events such as tardive dyskinesia, a consequence of risperidone therapy, as well as causing weight gain, gastrointestinal issues, and increased self-injurious and extreme behaviors that further worsen ASD symptoms [14]. Worsening of ASD symptoms and side effects due to prescription drug use may contribute to greater use of emergency departments and hospitalizations and higher costs of healthcare for adults with ASD [14, 70, 71].

\subsection{Strengths}

Our study is the first study to examine the extent of prescription drug use and types of polypharmacy among adults with ASD using a large population-based setting and realworld data. We used a three-state Medicaid database, a program that provides extensive pharmacy services and home- and community-based waivers to individuals with ASD [72, 73]. The current study is also the first study to estimate the average number of claims per year for specific therapeutic classes among adults with ASD and compare the rates with a matched sample of adults without ASD. State Medicaid programs have made consistent efforts to reduce prescription drug use and costs since the early 2000 s [74], and our study highlights that adults with ASD have substantial and/or off-label use of prescription drugs compared with other Medicaid beneficiaries.

\subsection{Limitations}

Despite the various strengths, our study was limited in some ways. Our study period was restricted and the definition of ASD and many drug-related policies/rebate programs may have changed after 2008. In addition, the list of drug classes coded using NDCs may not have been exhaustive and some drugs which are provided in intravenous form (e.g., anticancer therapies) or via an inpatient pharmacy are not reflected in the findings. We required adults with ASD to be continuously enrolled during the study period to allow their full prescription drug utilization dates to be captured, which could have excluded many individuals with sporadic Medicaid enrollment. We did not match the ASD cases and no-ASD controls on their enrollment period, which could have created certain baseline differences in the two groups. A majority of adults in the study were eligible by cash assistance, indicating that our sample was primarily low income and/or had high needs; thus, rates of drug use may be overrepresented for both ASD and no-ASD groups. Definitions of polypharmacy have varied substantially across studies [17, 18, 42], which may cause differential rates across studies. Comorbid diagnoses listed with an individuals' claim may not always be accurate or comprehensive. Due to the lack of information on diagnosis history or reasons for outpatient visits/hospitalizations, corroborating whether there was actual off-label prescription drug use was not possible.

\section{Conclusion}

Prescription drug use, general polypharmacy, and psychotropic polypharmacy among adults with ASD are significantly and substantially higher than in adults without ASD. Half of adults with ASD use six or more drug classes in a year and almost one-fifth of them use three or more psychotropic drug classes within a 90-day period. Higher prescription drug use among adults with ASD is not restricted to psychotropic drugs, but extends to prescriptions alleviating other medical needs including cardiovascular agents, statins, immunologics, gastrointestinal agents, antiparkinsonian agents, and many others. Older age, female gender, White race, and presence of three or more comorbid conditions among adults with ASD is significantly associated with using six or more prescription drug classes per year.

The study highlights considerable pharmaceutical needs of adults with ASD as well as the practice of off-label prescription use for adults with ASD by healthcare providers. A high prescription use among adults with ASD should be constantly monitored and physicians should play an active role in long-term treatment of ASD. In addition, further investigations about any use of alternative and complementary treatments that can affect a patient's response to core treatments should also be done. Long term use of prescription drugs may be associated with tolerance 
and sensitization which may worsen ASD core symptoms, and thus it is very critical to develop an optimum pharmacological management guideline of adult ASD interventions [75]. The issue of lack of evidence-based treatments and clinical trials should be addressed by healthcare research agencies, policymakers, and state health insurance programs.

\section{Compliance with Ethical Standards}

Informed consent Informed consent was not required for the current study which did not involve direct patient data collection. The study was exempted from the West Virginia Institutional Review Board. The research proposal for Medicaid database use was reviewed and successfully approved by Centers of Medicare and Medicaid Services under a data user agreement policy.

Funding Authors SM and US were partially funded by an IDeA-CTR award from the National Institute of General Medical Sciences, U54GM104942. The content is solely the responsibility of the authors and does not necessarily represent the official views of the NIH or the policy or position of West Virginia University (WVU) or any other affiliated organizations. All authors approved the final manuscript as submitted.

Conflict of interests All authors, including RV, SM, US, CS, SP, $\mathrm{ND}$, and MA, declare that there are no conflicts of interest for this research work.

Open Access This article is distributed under the terms of the Creative Commons Attribution-NonCommercial 4.0 International License (http://creativecommons.org/licenses/by-nc/4.0/), which permits any noncommercial use, distribution, and reproduction in any medium, provided you give appropriate credit to the original author(s) and the source, provide a link to the Creative Commons license, and indicate if changes were made.

\section{References}

1. Jang J, Dixon DR, Tarbox J, Granpeesheh D. Symptom severity and challenging behavior in children with ASD. Res Autism Spectr Disord. 2011;5(3):1028-32.

2. Matson JL, Wilkins J, Macken J. The relationship of challenging behaviors to severity and symptoms of autism spectrum disorders. J Mental Health Res Intellect Disabil. 2009;2(1):29-44.

3. Murphy GH, Beadle-Brown J, Wing L, Gould J, Shah A, Holmes $\mathrm{N}$. Chronicity of challenging behaviours in people with severe intellectual disabilities and/or autism: a total population sample. J Autism Dev Disord. 2005;35(4):405-18.

4. Matson JL, Rivet TT. Characteristics of challenging behaviours in adults with autistic disorder, PDD-NOS, and intellectual disability. J Intellect Dev Disabil. 2008;33(4):323-9.

5. Simonoff E, Pickles A, Charman T, Chandler S, Loucas T, Baird G. Psychiatric disorders in children with autism spectrum disorders: prevalence, comorbidity, and associated factors in a population-derived sample. J Am Acad Child Adolesc Psychiatry. 2008;47(8):921-9.

6. van Steensel FJ, Bogels SM, de Bruin EI. Psychiatric comorbidity in children with autism spectrum disorders: a comparison with children with ADHD. J Child Fam Stud. 2013;22(3):368-76.
7. Kohane IS, McMurry A, Weber G, MacFadden D, Rappaport L, Kunkel L, et al. The co-morbidity burden of children and young adults with autism spectrum disorders. PLoS One. 2012;7(4):e33224.

8. Buie T, Campbell DB, Fuchs GJ,3rd, Furuta GT, Levy J, Vandewater $\mathbf{J}$, et al. Evaluation, diagnosis, and treatment of gastrointestinal disorders in individuals with ASDs: a consensus report. Pediatrics. 2010;125(Suppl 1):S1-18.

9. McElhanon BO, McCracken C, Karpen S, Sharp WG. Gastrointestinal symptoms in autism spectrum disorder: a meta-analysis. Pediatrics. 2014;133(5):872-83.

10. LeClerc S, Easley D. Pharmacological therapies for autism spectrum disorder: a review. P T. 2015;40(6):389-97.

11. Volkmar F, Cook EH, Jr., Pomeroy J, Realmuto G, Tanguay P. Practice parameters for the assessment and treatment of children, adolescents, and adults with autism and other pervasive developmental disorders. Am Acad Child Adolesc Psychiatry Work Group Qual Issues. J Am Acad Child Adolesc Psychiatry. 1999;38(12 Suppl):32S-54S.

12. Baribeau DA, Anagnostou E. An update on medication management of behavioral disorders in autism. Curr Psychiatry Rep. 2014;16(3):437,014-0437-0.

13. Matson JL, Sipes M, Fodstad JC, Fitzgerald ME. Issues in the management of challenging behaviours of adults with autism spectrum disorder. CNS Drugs. 2011;25(7):597-606.

14. Myers SM. The status of pharmacotherapy for autism spectrum disorders. Expert Opin Pharmacother. 2007;8(11):1579-603.

15. Warren Z, Veenstra-VanderWeele J, Stone W, Bruzek JL, Nahmias AS, Foss-Feig JH, et al. Therapies for children with autism spectrum disorders. Comparative effectiveness reviews no. 26. Rockville: Agency for Healthcare Research and Quality; April 2011 Apr. Report No.: 11-EHC029-EF.

16. Fulton MM, Allen ER. Polypharmacy in the elderly: a literature review. J Am Acad Nurse Pract. 2005;17(4):123-32.

17. Frazier SC. Health outcomes and polypharmacy in elderly individuals: an integrated literature review. J Gerontol Nurs. 2005;31(9):4-11.

18. Bjerrum L, Rosholm JU, Hallas J, Kragstrup J. Methods for estimating the occurrence of polypharmacy by means of a prescription database. Eur J Clin Pharmacol. 1997;53(1):7-11.

19. Lake JK, Balogh R, Lunsky Y. Polypharmacy profiles and predictors among adults with autism spectrum disorders. Res Autism Spectr Disord. 2012;6(3):1142-9.

20. Dove D, Warren Z, McPheeters ML, Taylor JL, Sathe NA, Veenstra-VanderWeele J. Medications for adolescents and young adults with autism spectrum disorders: a systematic review. Pediatrics. 2012;130(4):717-26.

21. Esbensen AJ, Greenberg JS, Seltzer MM, Aman MG. A longitudinal investigation of psychotropic and non-psychotropic medication use among adolescents and adults with autism spectrum disorders. J Autism Dev Disord. 2009;39(9):1339-49.

22. Frazier TW, Shattuck PT, Narendorf SC, Cooper BP, Wagner M, Spitznagel EL. Prevalence and correlates of psychotropic medication use in adolescents with an autism spectrum disorder with and without caregiver-reported attention-deficit/hyperactivity disorder. J Child Adolesc Psychopharmacol. 2011;21(6):571-9.

23. Zito JM, Derivan AT, Kratochvil CJ, Safer DJ, Fegert JM, Greenhill LL. Off-label psychopharmacologic prescribing for children: history supports close clinical monitoring. Child Adolesc Psychiatry Ment Health. 2008;2(1):24.

24. Dalsgaard S, Nielsen HS, Simonsen M. Five-fold increase in national prevalence rates of attention-deficit/hyperactivity disorder medications for children and adolescents with autism spectrum disorder, attention-deficit/hyperactivity disorder, and other psychiatric disorders: a Danish register-based study. J Child Adolesc Psychopharmacol. 2013;23(7):432-9. 
25. Barnard-Brak L, Davis TN, Schmidt M, Richman DM. Effects associated with on- and off-label stimulant treatment of core autism and ADHD symptoms exhibited by children with autism spectrum disorder. Dev Neurorehabil. 2016;19(1):46-53.

26. Hirota $T$, Veenstra-Vanderweele J, Hollander E, Kishi $T$. Antiepileptic medications in autism spectrum disorder: a systematic review and meta-analysis. J Autism Dev Disord. 2014;44(4):948-57.

27. McCracken JT, McGough J, Shah B, Cronin P, Hong D, Aman $\mathrm{MG}$, et al. Risperidone in children with autism and serious behavioral problems. N Engl J Med. 2002;347(5):314-21.

28. Curran MP. Aripiprazole in the treatment of irritability associated with autistic disorder in paediatric patients: profile report. CNS Drugs. 2011;25(9):801-2.

29. Blankenship K, Erickson CA, Stigler KA, Posey DJ, McDougle CJ. Aripiprazole for irritability associated with autistic disorder in children and adolescents aged 6-17 years. Ped Health. 2010;4(4):375-81.

30. Owen R, Sikich L, Marcus RN, Corey-Lisle P, Manos G, McQuade RD, et al. Aripiprazole in the treatment of irritability in children and adolescents with autistic disorder. Pediatrics. 2009;124(6):1533-40.

31. Marcus RN, Owen R, Kamen L, Manos G, McQuade RD, Carson $\mathrm{WH}$, et al. A placebo-controlled, fixed-dose study of aripiprazole in children and adolescents with irritability associated with autistic disorder. J Am Acad Child Adolesc Psychiatry. 2009;48(11):1110-9.

32. Ching H, Pringsheim T. Aripiprazole for autism spectrum disorders (ASD). Cochrane Database Syst Rev. 2012;5:CD009043.

33. Adler BA, Wink LK, Early M, Shaffer R, Minshawi N, McDougle $\mathrm{CJ}$, et al. Drug-refractory aggression, self-injurious behavior, and severe tantrums in autism spectrum disorders: a chart review study. Autism. 2015;19(1):102-6.

34. Williams K, Wheeler DM, Silove N, Hazell P. Selective serotonin reuptake inhibitors (SSRIs) for autism spectrum disorders (ASD). Cochrane Database Syst Rev. 2010;8:CD004677.

35. West L, Brunssen SH, Waldrop J. Review of the evidence for treatment of children with autism with selective serotonin reuptake inhibitors. J Spec Pediatr Nurs. 2009;14(3):183-91.

36. Mandell DS, Morales KH, Marcus SC, Stahmer AC, Doshi J, Polsky DE. Psychotropic medication use among Medicaid-enrolled children with autism spectrum disorders. Pediatrics. 2008;121(3):e441-8.

37. Khanna R, Jariwala K, West-Strum D. Use and cost of psychotropic drugs among recipients with autism in a state Medicaid fee-for-service programme. $\mathbf{J}$ Intellect Disabil Res. 2013;57(2):161-71.

38. Rosenberg RE, Mandell DS, Farmer JE, Law JK, Marvin AR, Law PA. Psychotropic medication use among children with autism spectrum disorders enrolled in a national registry, 2007-2008. J Autism Dev Disord. 2010;40(3):342-51.

39. Spencer D, Marshall J, Post B, Kulakodlu M, Newschaffer C, Dennen T, et al. Psychotropic medication use and polypharmacy in children with autism spectrum disorders. Pediatrics. 2013;132(5):833-40.

40. Logan SL, Nicholas JS, Carpenter LA, King LB, Garrett-Mayer E, Charles JM. High prescription drug use and associated costs among Medicaid-eligible children with autism spectrum disorders identified by a population-based surveillance network. Ann Epidemiol. 2012;22(1):1-8.

41. Coury DL, Anagnostou E, Manning-Courtney P, Reynolds A, Cole L, McCoy R, et al. Use of psychotropic medication in children and adolescents with autism spectrum disorders. Pediatrics. 2012;130(Suppl 2):S69-76.

42. Zarowitz BJ, Stebelsky LA, Muma BK, Romain TM, Peterson EL. Reduction of high-risk polypharmacy drug combinations in patients in a managed care setting. Pharmacotherapy. 2005;25(11):1636-45.

43. Brager R, Sloand E. The spectrum of polypharmacy. Nurse Pract. 2005;30(6):44-50.

44. Faries D, Ascher-Svanum H, Zhu B, Correll C, Kane J. Antipsychotic monotherapy and polypharmacy in the naturalistic treatment of schizophrenia with atypical antipsychotics. BMC Psychiatry. 2005;27(5):26.

45. Chen H, Patel A, Sherer J, Aparasu R. The definition and prevalence of pediatric psychotropic polypharmacy. Psychiatr Serv. 2011;62(12):1450-5.

46. Jyrkka J, Enlund H, Korhonen MJ, Sulkava R, Hartikainen S. Polypharmacy status as an indicator of mortality in an elderly population. Drugs Aging. 2009;26(12):1039-48.

47. Essock SM, Covell NH, Leckman-Westin E, Lieberman JA, Sederer LI, Kealey E, et al. Identifying clinically questionable psychotropic prescribing practices for medicaid recipients in new york state. Psychiatr Serv. 2009;60(12):1595-602.

48. Mauch D, Pfefferle S, Booker C, Pustell M, Levin J. Report on state services to individuals with autism spectrum disorders (ASD). Centers for Medicare \& Medicaid Services (CMS) ASD Services Project: Centers for Medicare \& Medicaid Services (CMS); 2011. Report No.: S-10 CMS-33 No. 2.

49. Bruder MB, Kerins G, Mazzarella C, Sims J, Stein N. Brief report: the medical care of adults with autism spectrum disorders: identifying the needs. J Autism Dev Disord. 2012;42(11):2498504.

50. Oskoui M, Wolfson C. Treatment comfort of adult neurologists in childhood onset conditions. Can $J$ Neurol Sci. 2012;39(2):202-5.

51. Golnik A, Ireland M, Borowsky IW. Medical homes for children with autism: a physician survey. Pediatrics. 2009;123(3):966-71.

52. Miller SM. Nurses Caring for Adults with Autism in an Emergency Department: A Survey of Knowledge Doctoral Projects. http://scholarworks.sjsu.edu/etd_doctoral/7/. Accessed Jan 2016.

53. Buck TR, Viskochil J, Farley M, Coon H, McMahon WM, Morgan J, et al. Psychiatric Comorbidity and Medication Use in Adults with Autism Spectrum Disorder. J Autism Dev Disord. 2014

54. Croen LA, Zerbo O, Qian Y, Massolo ML, Rich S, Sidney S, et al. The health status of adults on the autism spectrum. Autism. 2015;19(7):814-23.

55. Schubart JR, Camacho F, Leslie D. Psychotropic medication trends among children and adolescents with autism spectrum disorder in the Medicaid program. Autism. 2014;18(6):631-7.

56. State HMO Penetration Rate, State Health Facts. http://kff.org/ other/state-indicator/hmo-penetration-rate/. Accessed Dec 2013.

57. Quality Resource Systems. Area Resource File. Fairfax: Health Resources and Services Administration, US Department of Health and Human Services, 2006.

58. Burke JP, Jain A, Yang W, Kelly JP, Kaiser M, Becker L, et al. Does a claims diagnosis of autism mean a true case? Autism. 2014;18(3):321-30.

59. Dodds L, Spencer A, Shea S, Fell D, Armson BA, Allen AC, et al. Validity of autism diagnoses using administrative health data. Chronic Dis Can. 2009;29(3):102-7.

60. Austin PC. An introduction to propensity score methods for reducing the effects of confounding in observational studies. Multivar Behav Res. 2011;46(3):399-424.

61. Clinical Classifications Software (CCS) for ICD-9-CM. Healthcare cost and Utilization Project. https://www.hcup-us.ahrq.gov/ toolssoftware/ccs/AppendixASingleDX.txt. Accessed June 2016.

62. HEDIS and Quality Measurement. HEDIS 2015 Final NDC Lists. http://www.ncqa.org/HEDISQualityMeasurement/HEDISMeasures/ HEDIS2015/HEDIS2015NDCLicense/HEDIS2015FinalNDCLists. aspx. Accessed June 2016. 
63. Oswald DP, Sonenklar NA. Medication use among children with autism spectrum disorders. J Child Adolesc Psychopharmacol. 2007; 17(3):348-55.

64. Starkstein S, Gellar S, Parlier M, Payne L, Piven J. High rates of parkinsonism in adults with autism. J Neurodev Disord. 2015;7(1):29.

65. Hollander E, Wang AT, Braun A, Marsh L. Neurological considerations: autism and Parkinson's disease. Psychiatry Res. 2009; 170(1):43-51.

66. Croen LA, Najjar DV, Ray GT, Lotspeich L, Bernal P. A comparison of health care utilization and costs of children with and without autism spectrum disorders in a large group-model health plan. Pediatrics. 2006;118(4):e1203-11.

67. 10 Year Timeline. https://www.autismspeaks.org/10year/ timeline. Accessed June 2016.

68. Arjun L, Hope C, Guyer J, Alker J. Medicaid and its role for children and youth with special health care needs (CYSHCN): a family perspective. Georgetown University, Health Policy Institute: Center for Children and Families/Family Voices; 2011.

69. Rubenstein E, Wiggins LD, Lee LC. A review of the differences in developmental, psychiatric, and medical endophenotypes between males and females with autism spectrum disorder. J Dev Phys Disabil. 2015;27(1):119-39.

70. Myer PA, Mannalithara A, Singh G, Singh G, Pasricha PJ, Ladabaum U. Clinical and economic burden of emergency department visits due to gastrointestinal diseases in the United States. Am J Gastroenterol. 2013;108(9):1496-507.

71. Kato K, Mikami K, Akama F, Yamada K, Maehara M, Kimoto K, et al. Clinical features of suicide attempts in adults with autism spectrum disorders. Gen Hosp Psychiatry. 2013;35(1):50-3.

72. Ruble LA, Heflinger CA, Renfrew JW, Saunders RC. Access and service use by children with autism spectrum disorders in Medicaid Managed Care. J Autism Dev Disord. 2005;35(1):3-13.

73. Semansky RM, Xie M, Mandell DS. Medicaid's increasing role in treating youths with autism spectrum disorders. Psychiatr Serv. 2011;62(6):588.

74. Recent Medicaid prescription drug laws and strategies. http:// www.ncsl.org/research/health/medicaid-pharmaceutical-lawsand-policies.aspx. Accessed June 2016.

75. Li M. Antipsychotic-induced sensitization and tolerance: behavioral characteristics, developmental impacts, and neurobiological mechanisms. J Psychopharmacol. 2016;30(8):749-70. 